Bull. Austral. Math. Soc.

$16 \mathrm{~A} 36,16 \mathrm{~A} 52,16 \mathrm{~A} 72$

Vol. $40(1989) \quad[109-111]$

\title{
A NOTE ON THE FIXED SUBRING OF AN FPF RING
}

\author{
JoHN ClaRK
}

\begin{abstract}
An associative ring $R$ with identity is called a left (right) FPF ring if given any finitely generated faithful left (right) $R$-module $A$ and any left (right) $R$-module $M$ then $M$ is the epimorphic image of a direct sum of copies of $A$. Faith and Page have asked if the subring of elements fixed by a finite group of automorphisms of an FPF ring need also be FPF. Here we present examples showing the answer to be negative in general.
\end{abstract}

\section{INTRODUCTION}

An associative ring $R$ with identity is said to be left (right) FPF (short for finitely pseudo-Frobenius) if every finitely generated faithful left (right) $R$-module generates the category of all left (right) $R$-modules, while $R$ is $F P F$ if it is both left and right $F P F$. Quasi-Frobenius rings, Prufer domains and self-injective commutative rings are all FPF. A recent monograph by Faith and Page [4] on FPF rings contains a list of fifteen open problems. Problem fourteen discusses the action of a finite group $G$ of automorphisms on a right $F P F$ ring $R$ and asks if in general the fixed ring $R^{G}=\{r \in R: \forall g \in G(g(r)=r)\}$ is also right $F P F$. Faith has shown in [2] that $R^{G}$ is $F P F$ when $R$ is commutative $F P F$ and finitely generated projective as a module over $R^{G}$. Here we provide two simple examples of FPF rings having commutative fixed rings which are not $F P F$.

\section{The EXamples}

For our first example we begin by letting $Q$ be the quaternion group of order eight, that is $Q=\left\langle a, b: a^{4}=b^{4}=1, a^{2}=b^{2}, b^{-1} a b=a^{-1}\right\rangle$. Then (see, for example Thomas and Wood [6]) $Q$ has automorphism group $S_{4}=\left\langle g, h: g^{4}=h^{2}=(g h)^{3}=1\right\rangle$ where $g(a)=a, g(b)=a b, h(a)=b$, and $h(b)=a$. Now form the group $\operatorname{ring} R=K[Q]$ where $K$ is the field of two elements. Then (see, for example, p.79 of Passman [5]), since $Q$ is finite, $R$ is self-injective. Thus, since $R$ is Artinian, $R$ is quasi-Frobenius and so $F P F$. Now let $G$ denote the group of automorphisms of $R$ obtained by extending linearly to $R$ the action of $S_{4}$ on $Q$. Then a straightforward calculation shows that

$$
R^{G}=\left\{0,1, a^{2}, 1+a^{2}, w, 1+w, a^{2}+w, 1+a^{2}+w\right\}
$$

\section{Received 4 October 1988}

This paper was written while the author was visiting the Department of Mathematics and Statistics at the University of Idaho. He is most grateful for the hospitality shown to him by the members of the Department and in particular Willy Brandal.

Copyright Clearance Centre, Inc. Serial-fee code: 0004-9729/89 \$A2.00+0.00. 
where $w=a+a^{3}+b+a b+a^{2} b+a^{3} b$. Moreover $R^{G}$ is commutative and its proper nonzero ideals are just $I=\left\{0,1+a^{2}\right\}, J=\{0, w\}, K=\left\{0,1+a^{2}+w\right\}$ and the unique maximal ideal $M=\left\{0,1+a^{2}, w, 1+a^{2}+w\right\}$. Also $M^{2}=0$ and, since $R^{G}$ is Artinian, $R^{G}$ is its own classical ring of quotients.

Now Theorem A of [3] implies that the classical quotient ring of a commutative $F P F$ ring is self-injective. Thus to show that $R^{G}$ is not $F P F$ it suffices to show that it is not self-injective. To this end we define $f: I \rightarrow J$ by $f\left(1+a^{2}\right)=w$. Then $f$ is an $R^{G}$-homomorphism, well-defined since $M^{2}=0$, which cannot be extended to an endomorphism on $R^{G}$ since $I \cap J=0$. Thus, by Baer's criterion for injectivity, $R^{G}$ is not self-injective and so not $F P F$.

Our second example is commutative and of arbitrary prime characteristic $p$. Let $K$ be a field of characteristic $p$ and form

$$
R=K\left[\left[x_{1}, \ldots, x_{p}\right]\right] / J
$$

where $x_{1}, \ldots, x_{p}$ are commuting indeterminates and $J$ is the ideal of the power series ring generated by $x_{i}^{2}-x_{j}^{2}$ and $x_{i} x_{j}$ for $i \neq j$ and $i, j \in\{1, \ldots, p\}$. Then $R$ is a commutative local quasi-Frobenius ring and an arbitrary element of $R$ has expression

$$
a+b_{1} x_{1}+b_{2} x_{2}+\ldots+b_{p} x_{p}+c x_{1}^{2} \text {, where } a, b_{1}, b_{2}, \ldots, b_{p}, c \in K \text {. }
$$

Now let $g$ be the automorphism of $R$ determined by $g\left(x_{i}\right)=x_{i+1}$ for $i=1, \ldots, p-1$ and $g\left(x_{p}\right)=x_{1}$. Then $G=\langle g\rangle$ is of order $p$ and $R^{G}$ consists of elements of the form $\left(^{*}\right)$ where $b_{1}=b_{2}=\ldots=b_{p}$. Noting that the units of $R$ are those with nonzero $a$, straightforward arguments show that, as for our first example, $R^{G}$ is commutative local Artinian but not $F P F$, having the property that the nonzero ideals properly contained in the maximal ideal are all simple. Indeed, taking $p=2$ and $K$ as the field of two elements gives the same $R^{G}$ (but smaller $R$, of order 16) as before. In fact this is a minimal counterexample.

Finally we remark that the ring $R$ of our first example was used in [1] as an example of an FPF ring whose centre $C$, of order 32 , is not $F P F$. Replacing $G=S_{4}$ by the inner automorphism group $H$ on $Q$ and identifying this with its linear extension to $R$ gives $R^{H}=C$, thus providing yet another example.

\section{REFERENCES}

[1] J. Clark, 'The centre of an FPF ring need not be FPF', Bull. Austral. Math. Soc. 37 (1988), 235-236.

[2] C. Faith, 'Subrings of self-injective and FPF rings', in Advances in noncommutative ring theory: Lecture Notes in Math. 951, pp. 12-20 (Springer-Verlag, Berlin-New York, 1982).

[3] C. Faith, Injective modules and injective quotient rings: Lecture Notes in Pure and Applied Mathematics 72 (Marcel Dekker, Inc., New York, 1982). 
[4] C. Faith and S. Page, FPF Ring Theory. Faithful modules and generators of mod-R: London Math. Soc. Lecture Notes 88 (Cambridge University Press, Cambridge, London, New York, New Rochelle, Melbourne, Sydney, 1984).

[6] D.S. Passman, The algebraic structure of group rings (Pure Appl. Math., Wiley-Interscience, New York, London, Sydney, 1977).

[6] A.D. Thomas and G.V. Wood, Group tables (Shiva Publishing Ltd, Orpington, 1980).

Department of Mathematics and Statistics

University of Otago

Dunedin

New Zealand 